\title{
Robin Boundary Value Problem Depending on Parameters in a Ring Domain
}

\author{
İlker Gençtürk
}

Department of Mathematics, Faculty of Science and Arts, Kırıkkale University, Kırıkkale, Turkey

\section{Article Info}

Keywords: Cauchy-Riemann operator, Ring domain, Robin problem 2010 AMS: 30E25, 31 A10

Received: 15 September 2020

Accepted: 27 November 2020

Available online: 15 December 2020

\begin{abstract}
This study is devoted to give solvability conditions and solutions of the Robin boundary problem with constant coefficients for the homogeneous and the inhomogeneous CauchyRiemann equation in an annular domain. In order to get results, known representations and theorems in the literature are used. The representations for the solutions and solvability conditions are given in explicit form and here only a special Robin problem is considered. At the end of the paper, it is concluded that with some choices, boundary value problems for the Cauchy-Riemann equation reduce to some basic boundary problems in the ring domain.
\end{abstract}

\section{Introduction and preliminaries}

Recently, some complex model partial differential equations, which have important applications in some areas of applied sciences, were investigated in detail, especially for Robin problem see [1]-[7]. Also, the solvability and solutions of complex partial differential equations with boundary conditions were considered by many mathematicians. [8]-[11].

The Robin problem, called as third boundary problem, is a mixed form of the Dirichlet and the Neumann problems, which are basic boundary value problems in complex analysis.

The main aim of this paper is to give solvability conditions and solutions of Robin problem with real parameters for CauchyRiemann operators in an annular domain $R=\{z \in \mathbb{C}: 0<r<|z|<1\}$. The results in this paper are obtained by using some integral representations in the annular domain [12]-[14], which are similar to ones in the unit disc. [15, 16].

For the convenience of the reader, we recall some relevant theorems without proofs:

Theorem 1.1 (The Complex Form of Gauss Theorem). [15] Let $D \subset \mathbb{C}$ be a bounded domain with smooth boundary $\partial D$, and the closure $\bar{D}=D \cup \partial D$. Assume that $w \in C^{1}(D ; \mathbb{C}) \cap C(\bar{D} ; \mathbb{C})$. Then

$$
\int_{D} w_{\bar{z}}(z) d x d y=\frac{1}{2 i} \int_{\partial D} w(z) d z, \int_{D} w_{z}(z) d x d y=-\frac{1}{2 i} \int_{\partial D} w(z) d \bar{z}
$$

where

$$
\partial_{z}=\frac{1}{2}\left(\frac{\partial}{\partial x}-i \frac{\partial}{\partial y}\right), \partial_{\bar{z}}=\frac{1}{2}\left(\frac{\partial}{\partial x}+i \frac{\partial}{\partial y}\right) ; z=x+i y, x, y \in \mathbb{R}
$$


Theorem 1.2 (Cauchy Integral Formula). Let $\gamma$ be a simply closed smooth curve and $D$ be the inner domain, bounded by $\gamma$. If $w$ is an analytic function in $D$, continuous in $\bar{D}$ and $z \in D$, then

$$
w(z)=\frac{1}{2 \pi i} \int_{\gamma} w(\zeta) \frac{d \zeta}{\zeta-z} .
$$

Theorem 1.3 (Cauchy-Pompeiu representation). [17] Under the assumptions of Theorem 1.1, we have for $z \in D$ that

$$
w(z)=\frac{1}{2 \pi i} \int_{\partial D} w(\zeta) \frac{d \zeta}{\zeta-z}-\frac{1}{\pi} \int_{D} w_{\bar{\zeta}}(\zeta) \frac{d \xi d \eta}{\zeta-z}
$$

where $\zeta=\xi+i \eta$.

The Dirichlet boundary value problem for analytic functions in $R$ is

$$
w_{\bar{z}}=0, w=\gamma \text { on } \partial R
$$

for a given function $\gamma \in C(\partial \mathbb{R}, \mathbb{C})$.

The following theorem is proved in [14]:

Theorem 1.4. The Dirichlet problem (1.2) is solvable if and only if for $z \in R$

$$
\frac{1}{2 \pi i} \int_{\partial R} \gamma(\zeta) \frac{\bar{z} d \zeta}{1-\bar{z} \zeta}=\frac{1}{2 \pi i} \int_{\partial R} \gamma(z) \frac{\bar{z} d \zeta}{r^{2}-\bar{z} \zeta}=0
$$

in the class of analytic functions. Then the unique solution is given by the Cauchy type integral

$$
w(z)=\frac{1}{2 \pi i} \int_{\partial R} \gamma(\zeta) \frac{d \zeta}{\zeta-z}
$$

The normal derivative on the boundary of $R$ is defined by

$$
\partial_{v}=\left\{\begin{array}{c}
z \partial_{z}+\bar{z} \partial_{\bar{z}},|z|=1, \\
-\frac{z}{r} \partial_{z}-\frac{\bar{z}}{r} \partial_{\bar{z}},|z|=r .
\end{array}\right.
$$

The Robin boundary value problem for analytic functions in $R$ is

$$
w_{\bar{z}}=0, w+\lambda|z| \partial_{\nu} w=\gamma \quad \text { on } \quad \partial R, \lambda= \begin{cases}1, & |z|=1, \\ -1, & |z|=r\end{cases}
$$

for a given function $\gamma \in C(\partial R, \mathbb{C})$.

\section{The Robin boundary value problem depending on parameters for analytic functions}

In this section, in $R$ we investigate for $\alpha, \beta \in \mathbb{R}$, and $\gamma \in C(\partial R, \mathbb{C})$, the Robin boundary problem

$$
\begin{array}{r}
w_{\bar{z}}=0, z \in R \\
\left(\alpha w+\beta \lambda|z| \partial_{\nu} w\right)=\gamma, z \in \partial R .
\end{array}
$$

As a consequence of analyticity of $w$, the boundary condition (2.2) can be rewritten in the form

$$
\left.\left(\alpha w+\beta z w_{z}\right)\right|_{\partial R}=\gamma .
$$

Introducing a new function

$$
\varphi=\alpha w+\beta z w_{z}
$$


the boundary problem (2.1)-(2.2) turns out as the Dirichlet problem

$$
\varphi_{\bar{z}}=0 \text { in } R, \varphi=\gamma \text { on } \partial R
$$

On account of Theorem 1.4, boundary problem (2.3) can be uniquely solved if and only if for $z \in R$, the function $\gamma$ satisfies that

$$
\frac{1}{2 \pi i} \int_{\partial R} \gamma(\zeta) \frac{\bar{z} d \zeta}{1-\bar{z} \zeta}=\frac{1}{2 \pi i} \int_{\partial R} \gamma(z) \frac{\bar{z} d \zeta}{r^{2}-\bar{z} \zeta}=0
$$

Then the unique solution of the problem (2.3) is obtained as

$$
\varphi(z)=\frac{1}{2 \pi i} \int_{\partial R} \gamma(\zeta) \frac{d \zeta}{\zeta-z}
$$

We note that as an analytic function in $R, w(z)$ has a unique representation by a Laurent series

$$
w(z)=\sum_{n=-\infty}^{\infty} c_{n} z^{n}
$$

which converges in $R$.

Then, we have

$$
\begin{aligned}
\varphi(z) & =\alpha w(z)+\beta z w_{z}(z) \\
& =\alpha \sum_{n=-\infty}^{\infty} c_{n} z^{n}+\beta \sum_{n=-\infty}^{\infty} n c_{n} z^{n} \\
& =\sum_{n=-\infty}^{\infty}(\alpha+n \beta) c_{n} z^{n} .
\end{aligned}
$$

Considering (2.5), it yields

$$
\begin{aligned}
\sum_{n=-\infty}^{\infty}(\alpha+n \beta) c_{n} z^{n} & =\frac{1}{2 \pi i} \int_{\partial R} \gamma(\zeta) \frac{d \zeta}{\zeta-z} \\
& =\sum_{n=0}^{\infty} \frac{1}{2 \pi i} \int_{|\zeta|=1} \gamma(\zeta) \frac{d \zeta}{\zeta^{n+1}} z^{n}+\sum_{n=-\infty}^{-1} \frac{1}{2 \pi i} \int_{|\zeta|=r} \gamma(\zeta) \frac{d \zeta}{\zeta^{n+1}} z^{n}
\end{aligned}
$$

Comparing coefficients of both sides of (2.6), we have as long as $\alpha+n \beta \neq 0$,

$$
\begin{aligned}
c_{n} & =\frac{1}{\alpha+n \beta} \frac{1}{2 \pi i} \int_{|\zeta|=1} \gamma(\zeta) \frac{d \zeta}{\zeta^{n+1}}, n=0,1,2, \ldots \\
c_{n} & =\frac{1}{\alpha+n \beta} \frac{1}{2 \pi i} \int_{|\zeta|=r} \gamma(\zeta) \frac{d \zeta}{\zeta^{n+1}}, n=\ldots,-2,-1 .
\end{aligned}
$$

Therefore, we can assert that

$$
w(z)=\sum_{n=0}^{\infty} \frac{1}{\alpha+n \beta}\left(\frac{1}{2 \pi i} \int_{|\zeta|=1} \gamma(\zeta) \frac{d \zeta}{\zeta^{n+1}}\right) z^{n}+\sum_{n=-\infty}^{-1} \frac{1}{\alpha+n \beta}\left(\frac{1}{2 \pi i} \int_{|\zeta|=r} \gamma(\zeta) \frac{d \zeta}{\zeta^{n+1}}\right) z^{n}
$$

Theorem 2.1. For $\alpha, \beta \in \mathbb{R}$, and $\gamma \in C(\partial R, \mathbb{C})$, Robin boundary value problem (2.1)-(2.2) in $R$ is solvable if and only if condition (2.4) is satisfied. In this case, solution of the problem if $\alpha+n \beta \neq 0$ for all $n \in \mathbb{Z}$ is given by (2.7). 


\section{The Robin boundary value problem depending on parameters for inhomogeneous Cauchy- Riemann equation}

In this section, we deal for $\alpha, \beta \in \mathbb{R}, \gamma \in C(\partial R, \mathbb{C})$ and $f \in C^{a}(\bar{R} ; \mathbb{C}), 0<a<1$, with the Robin boundary problem

$$
\begin{array}{r}
w_{\bar{z}}=f, z \in R, \\
\left(\alpha w+\beta \lambda|z| \partial_{\nu} w\right)=\gamma, z \in \partial R .
\end{array}
$$

Solutions of equation $w_{\bar{z}}=f$ have the form

$$
w(z)=\varphi(z)-\frac{1}{\pi} \int_{R} \frac{f(\zeta)}{\zeta-z} d \xi d \eta
$$

where $\varphi(z)$ is any analytic function in $R$, see [17].

By differentiating with respect to $z$ implies

$$
w_{z}=\varphi_{z}-\frac{1}{\pi} \int_{R} \frac{f(\zeta)}{(\zeta-z)^{2}} d \xi d \eta
$$

We note that the latter derivative is taken in distributional sense, see [15].

By introducing the new function

$$
\varphi=w+\frac{1}{\pi} \int_{R} \frac{f(\zeta)}{\zeta-z} d \xi d \eta
$$

and using $w_{\bar{z}}=f$, the problem (3.1)-(3.2) is reduced to

$$
\begin{aligned}
\varphi_{\bar{z}} & =0, \text { in } R \\
\left(\alpha \varphi+\beta z \varphi_{z}\right) & =\left(\gamma+\frac{1}{\pi} \int_{R}\left[\frac{\beta z}{(\zeta-z)^{2}}-\frac{\alpha}{z-\zeta}\right] f(\zeta) d \xi d \eta-\beta \bar{z} f\right):=\widehat{\gamma}, \text { on } \partial R
\end{aligned}
$$

the Robin problem in the previous section. By Theorem 2.1, (3.4)-(3.5) is solvable if and only if

$$
\frac{1}{2 \pi i} \int_{\partial R} \widehat{\gamma}(\zeta) \frac{\bar{z} d \zeta}{1-\bar{z} \zeta}=\frac{1}{2 \pi i} \int_{\partial R} \widehat{\gamma}(z) \frac{\bar{z} d \zeta}{r^{2}-\bar{z} \zeta}=0
$$

In this case, solution of the problem if $\alpha+n \beta \neq 0$ for all $n \in \mathbb{Z}$ is given by

$$
\varphi(z)=\sum_{n=0}^{\infty} \frac{1}{\alpha+n \beta}\left(\frac{1}{2 \pi i} \int_{|\zeta|=1} \widehat{\gamma}(\zeta) \frac{d \zeta}{\zeta^{n+1}}\right) z^{n}+\sum_{n=-\infty}^{-1} \frac{1}{\alpha+n \beta}\left(\frac{1}{2 \pi i} \int_{|\zeta|=r} \widehat{\gamma}(\zeta) \frac{d \zeta}{\zeta^{n+1}}\right) z^{n}
$$

It is clear that (3.5) for $z \in \partial R$ is equal to

$$
\widehat{\gamma}(z)=\gamma(z)+\frac{1}{\pi} \int_{R}\left[\frac{\beta z}{(\zeta-z)^{2}}-\frac{\alpha}{z-\zeta}\right] f(\zeta) d \xi d \eta-\beta \bar{z} f(z)
$$

So, by (3.8), the first boundary integral in (3.6) for $t=t_{1}+i t_{2}$ can be written as

$$
\frac{1}{2 \pi i} \int_{\partial R} \widehat{\gamma}(\zeta) \frac{\bar{z} d \zeta}{1-\bar{z} \zeta}=\frac{1}{2 \pi i} \int_{\partial R}\left(\gamma(\zeta)+\frac{1}{\pi} \int_{R}\left[\frac{\beta \zeta}{(\zeta-t)^{2}}-\frac{\alpha}{\zeta-t}\right] f(t) d t_{1} d t_{2}-\beta \bar{\zeta} f(\zeta)\right) \frac{\bar{z} d \zeta}{1-\bar{z} \zeta}
$$

By applying Fubini's theorem when changing the order of integrations, we obtain 


$$
\begin{aligned}
\frac{1}{2 \pi i} \int_{\partial R} \widehat{\gamma}(\zeta) \frac{\bar{z} d \zeta}{1-\bar{z} \zeta}= & \frac{1}{2 \pi i} \int_{\partial R} \gamma(\zeta) \frac{\bar{z} d \zeta}{1-\bar{z} \zeta}+\frac{1}{\pi} \int_{R} f(t)\left(\frac{1}{2 \pi i} \int_{\partial R}\left[\frac{\beta \zeta}{(\zeta-t)^{2}}-\frac{\alpha}{\zeta-t}\right] \frac{\bar{z} d \zeta}{1-\bar{z} \zeta}\right) d t_{1} d t_{2} \\
& -\frac{1}{2 \pi i} \int_{\partial R} \beta \bar{\zeta} f(\zeta) \frac{\bar{z} d \zeta}{1-\bar{z} \zeta}
\end{aligned}
$$

By aid of the Cauchy integral formula (1.1),

$$
\frac{1}{2 \pi i} \int_{\partial R} \frac{\beta \zeta}{(\zeta-t)^{2}} \frac{d \zeta}{1-\bar{z} \zeta}=\frac{1}{2 \pi i} \int_{|\zeta|=1} \frac{\beta \zeta}{(\zeta-t)^{2}} \frac{d \zeta}{1-\bar{z} \zeta}-\frac{1}{2 \pi i} \int_{|\zeta|=r} \frac{\beta \zeta}{(\zeta-t)^{2}} \frac{d \zeta}{1-\bar{z} \zeta}=\frac{\beta}{(1-\bar{z} t)^{2}}
$$

and

$$
\frac{1}{2 \pi i} \int_{\partial R} \frac{\alpha}{\zeta-t} \frac{d \zeta}{1-\bar{z} \zeta}=\frac{1}{2 \pi i} \int_{|\zeta|=1} \frac{\alpha}{\zeta-t} \frac{d \zeta}{1-\bar{z} \zeta}-\frac{1}{2 \pi i} \int_{|\zeta|=r} \frac{\alpha}{\zeta-t} \frac{d \zeta}{1-\bar{z} \zeta}=\frac{\alpha}{1-\bar{z} t},
$$

hence it can be shown that

$$
\frac{1}{2 \pi i} \int_{\partial R} \widehat{\gamma}(\zeta) \frac{\bar{z} d \zeta}{1-\bar{z} \zeta}=\frac{1}{2 \pi i} \int_{\partial R}[\gamma(\zeta)-\beta \bar{\zeta} f(\zeta)] \frac{\bar{z} d \zeta}{1-\bar{z} \zeta}+\frac{1}{\pi} \int_{R} \bar{z} f(\zeta) \frac{\beta-\alpha+\alpha \bar{z} \zeta}{(1-\bar{z} \zeta)^{2}} d \xi d \eta=0
$$

With similar calculations, for the second boundary integral in (3.6), we obtain

$$
\frac{1}{2 \pi i} \int_{\partial R} \widehat{\gamma}(\zeta) \frac{\bar{z} d \zeta}{r^{2}-\bar{z} \zeta}=\frac{1}{2 \pi i} \int_{\partial R}[\gamma(\zeta)-\beta \bar{\zeta} f(\zeta)] \frac{\bar{z} d \zeta}{r^{2}-\bar{z} \zeta}+\frac{1}{\pi} \int_{R} \bar{z} f(\zeta) \frac{r^{2}(\beta-\alpha)+\alpha \bar{z} \zeta}{\left(r^{2}-\bar{z} \zeta\right)^{2}} d \xi d \eta=0
$$

If the value of (3.8) is substituted in (3.7), we can get for $\frac{\alpha}{\beta} \notin \mathbb{Z}$,

$$
\begin{aligned}
\varphi(z)= & \sum_{n=0}^{\infty} \frac{1}{\alpha+n \beta}\left(\frac{1}{2 \pi i} \int_{|\zeta|=1}\left(\gamma(\zeta)+\frac{1}{\pi} \int_{R}\left[\frac{\beta \zeta}{(\zeta-t)^{2}}-\frac{\alpha}{\zeta-t}\right] f(t) d t_{1} d t_{2}-\beta \bar{\zeta} f(\zeta)\right) \frac{d \zeta}{\zeta n+1}\right) z^{n} \\
& +\sum_{n=-\infty}^{-1} \frac{1}{\alpha+n \beta}\left(\frac{1}{2 \pi i} \int_{|\zeta|=r}\left(\gamma(\zeta)+\frac{1}{\pi} \int_{R}\left[\frac{\beta \zeta}{(\zeta-t)^{2}}-\frac{\alpha}{\zeta-t}\right] f(t) d t_{1} d t_{2}-\beta \bar{\zeta} f(\zeta)\right) \frac{d \zeta}{\zeta n+1}\right) z^{n}
\end{aligned}
$$

or equivalently

$$
\begin{gathered}
\varphi(z)=\sum_{n=0}^{\infty} \frac{1}{\alpha+n \beta}\left(\frac{1}{2 \pi i} \int_{|\zeta|=1} \gamma(\zeta) \frac{d \zeta}{\zeta^{n+1}}+\frac{1}{2 \pi i} \int_{|\zeta|=1}\left(\frac{1}{\pi} \int_{R} f(t)\left[\frac{\beta \zeta}{(\zeta-t)^{2}}-\frac{\alpha}{\zeta-t}\right] d t_{1} d t_{2}\right) \frac{d \zeta}{\zeta^{n+1}}\right. \\
\left.\quad-\frac{1}{2 \pi i} \int_{|\zeta|=1} \beta \bar{\zeta} f(\zeta) \frac{d \zeta}{\zeta n+1}\right) z^{n} \\
+\sum_{n=-\infty}^{-1} \frac{1}{\alpha+n \beta}\left(\frac{1}{2 \pi i} \int_{|\zeta|=r} \gamma(\zeta) \frac{d \zeta}{\zeta^{n+1}}+\frac{1}{2 \pi i} \int_{|\zeta|=r}\left(\frac{1}{\pi} \int_{R} f(t)\left[\frac{\beta \zeta}{(\zeta-t)^{2}}-\frac{\alpha}{\zeta-t}\right] d t_{1} d t_{2}\right) \frac{d \zeta}{\zeta^{n+1}}\right. \\
\left.-\frac{1}{2 \pi i} \int_{|\zeta|=r} \beta \bar{\zeta} f(\zeta) \frac{d \zeta}{\zeta^{n+1}}\right) z^{n} .
\end{gathered}
$$

Because of

$$
\frac{1}{2 \pi i} \int_{|\zeta|=1}\left(\frac{1}{\pi} \int_{R} f(t)\left[\frac{\beta \zeta}{(\zeta-t)^{2}}-\frac{\alpha}{\zeta-t}\right] d t_{1} d t_{2}\right) \frac{d \zeta}{\zeta^{n+1}}=0, \text { for } n=0,1, . .
$$


and

$$
\frac{1}{2 \pi i} \int_{|\zeta|=r}\left(\frac{1}{\pi} \int_{R} f(t)\left[\frac{\beta \zeta}{(\zeta-t)^{2}}-\frac{\alpha}{\zeta-t}\right] d t_{1} d t_{2}\right) \frac{d \zeta}{\zeta^{n+1}}=0, \text { for } n=\ldots,-2,-1
$$

we get

$$
\begin{aligned}
\varphi(z)= & \sum_{n=0}^{\infty} \frac{1}{\alpha+n \beta}\left(\frac{1}{2 \pi i} \int_{|\zeta|=1}(\gamma(\zeta)-\beta \bar{\zeta} f(\zeta)) \frac{d \zeta}{\zeta^{n+1}}\right) z^{n} \\
& +\sum_{n=-\infty}^{-1} \frac{1}{\alpha+n \beta}\left(\frac{1}{2 \pi i} \int_{|\zeta|=r}(\gamma(\zeta)-\beta \bar{\zeta} f(\zeta)) \frac{d \zeta}{\zeta^{n+1}}\right) z^{n}
\end{aligned}
$$

By using (3.3), solution of the problem (3.1)-(3.2) can be found as

$$
\begin{aligned}
w(z)= & \sum_{n=0}^{\infty} \frac{1}{\alpha+n \beta}\left(\frac{1}{2 \pi i} \int_{|\zeta|=1}(\gamma(\zeta)-\beta \bar{\zeta} f(\zeta)) \frac{d \zeta}{\zeta n+1}\right) z^{n} \\
& +\sum_{n=-\infty}^{-1} \frac{1}{\alpha+n \beta}\left(\frac{1}{2 \pi i} \int_{|\zeta|=r}(\gamma(\zeta)-\beta \bar{\zeta} f(\zeta)) \frac{d \zeta}{\zeta^{n+1}}\right) z^{n} \\
& +\frac{1}{\pi} \int_{R} \frac{1}{z-\zeta} f(\zeta) d \xi d \eta
\end{aligned}
$$

Finally, we have just proved the following:

Theorem 3.1. For $\alpha, \beta \in \mathbb{R}, f \in C^{a}(\bar{R} ; \mathbb{C}), 0<a<1, \gamma \in C(\partial R ; \mathbb{C})$, the Robin problem

$$
w_{\bar{z}}=f \text { in } R, \alpha w+\beta \lambda|z| \partial_{v} w=\gamma \text { on } \partial R
$$

is solvable if and only if for all $z \in R$

$$
\frac{1}{2 \pi i} \int_{\partial R}[\gamma(\zeta)-\beta \bar{\zeta} f(\zeta)] \frac{\bar{z} d \zeta}{1-\bar{z} \zeta}+\frac{1}{\pi} \int_{R} \bar{z} f(\zeta) \frac{\beta-\alpha+\alpha \bar{z} \zeta}{(1-\bar{z} \zeta)^{2}} d \xi d \eta=0
$$

and

$$
\frac{1}{2 \pi i} \int_{\partial R}[\gamma(\zeta)-\beta \bar{\zeta} f(\zeta)] \frac{\bar{z} d \zeta}{r^{2}-\bar{z} \zeta}+\frac{1}{\pi} \int_{R} \bar{z} f(\zeta) \frac{r^{2}(\beta-\alpha)+\alpha \bar{z} \zeta}{\left(r^{2}-\bar{z} \zeta\right)^{2}} d \xi d \eta=0
$$

Then, the solution of the problem if $\alpha+n \beta \neq 0$ for all $n \in \mathbb{Z}$ is represented by

$$
\begin{aligned}
w(z)= & \sum_{n=0}^{\infty} \frac{1}{\alpha+n \beta}\left(\frac{1}{2 \pi i} \int_{|\zeta|=1}(\gamma(\zeta)-\beta \bar{\zeta} f(\zeta)) \frac{d \zeta}{\zeta^{n+1}}\right) z^{n} \\
& +\sum_{n=-\infty}^{-1} \frac{1}{\alpha+n \beta}\left(\frac{1}{2 \pi i} \int_{|\zeta|=r}(\gamma(\zeta)-\beta \bar{\zeta} f(\zeta)) \frac{d \zeta}{\zeta^{n+1}}\right) z^{n} \\
& +\frac{1}{\pi} \int_{R} \frac{1}{z-\zeta} f(\zeta) d \xi d \eta
\end{aligned}
$$




\section{Conclusion}

In this paper, a special kind of Robin problem for analytic functions (Theorem 2.1) and more generally for the inhomogeneous Cauchy-Riemann equation (Theorem 3.1) are investigated in a concentric ring domain. The representations of the solutions and solvability conditions are aimed for in explicit form.

Let us reconsider the Robin boundary condition

$$
\left(\alpha w+\beta \lambda|z| \partial_{v} w\right)=\gamma \text { on } R
$$

Under above boundary condition (4.1), with some special cases of $\alpha$ and $\beta$, the following results can be obtained:

i.) By choosing $\alpha=\beta=1$, we have $\left(w+\lambda|z| \partial_{v} w\right)=\gamma$ on $R$. In this case, in (2.7), the coefficient of $z^{-1}, c_{-1}$ may take arbitrary values from $\mathbb{C}$. Hence, for solvability of the problem, the condition $\frac{1}{2 \pi i} \int_{|\zeta|=r} \gamma(\zeta) d \zeta=0$ is needed. Furthermore, with an additional condition $z_{0} w\left(z_{0}\right)=c$, for some fixed point $z_{0} \in R, c \in \mathbb{C}$, the problem is uniquely solvable. This problem is another special kind of Robin problem and appears as Theorem 2.2.14 (for analytic functions) in [14]. As is the analytic case, by applying similar arguments, in the inhomogeneous case, the conditions $\frac{1}{2 \pi i} \int_{|\zeta|=r}(\gamma(\zeta)-\bar{\zeta} f(\zeta)) d \zeta=0$ (for solvability) and $z_{0} w\left(z_{0}\right)=c$, for some fixed point $z_{0} \in R, c \in \mathbb{C}$ (for uniqueness of the solution) are needed. [14, Theorem 2.3.18 ]

ii.) By choosing $\alpha=1$ and $\beta=0$, we have $w=\gamma$ on $\partial R$. Hence, these problems are reduced to the Dirichlet problems for analytic functions and the inhomogeneous Cauchy-Riemann equation, respectively, in [14, Theorem 2.2.12 and Theorem 2.3.16].

iii.) By choosing $\alpha=0$, and $\beta=1$, we have $\left(\lambda|z| \partial_{\nu} w\right)=\gamma$ on $R$. Hence, these problems are reduced to the Neumann problems for analytic functions and the inhomogeneous Cauchy-Riemann equation, respectively in [14, Theorem 2.2.13 and Theorem 2.3.17], with additional conditions.

\section{Acknowledgement}

We would like to thank the referees for carefully reading our manuscript and for giving such constructive comments which substantially helped improving the quality of our paper.

\section{References}

[1] J. Diaz, V. Péron, Equivalent Robin boundary conditions for acoustic and elastic media, Math. Models Methods Appl. Sci., 26(08) (2016), $1531-1566$.

[2] B. Gustafsson, A. Vasil'ev, Conformal and Potential Analysis in Hele-Shaw Cells, Springer Science Business Media, 2006.

[3] K. Gustafson, T. Abe, The third boundary condition-was it Robin's?, Math. Intelligencer, 20 (1998), 63-71.

[4] Y. R. Linares, C. J. Vanegas, A Robin boundary value problem in the upper half plane for the Bitsadze equation, J. Math. Anal. Appl., 419(1) (2014), 200-217.

[5] G. Lozada-Cruz, C. E. Rubio-Mercedes, J. Rodrigues-Ribeiro, Numerical solution of heat equation with singular Robin boundary condition, TEMA Tend. Mat. Apl. Comput., 19(2) (2018), 209-220.

[6] D. Medková, P. Krutitskii, Neumann and Robin problems in a cracked domain with jump conditions on cracks, J. Math. Anal. Appl., 301(1) (2005), 99-114.

[7] R. Novák, Bound states in waveguides with complex Robin boundary conditions, Asymptot. Anal., 96(3-4) (2016), 251-281.

[8] H. Begehr, E. A. Gaertner, Dirichlet problem for the inhomogeneous polyharmonic equation in the upper half plane, Georgian Math. J., 14(1) (2007),

[9] H3-52.

[10] İ. Gençtürk, K. Koca, Dirichlet boundary value problem for an $n^{\text {th }}$ order complex partial differential equation, Gen. Math., 23(1-2) (2015), 39-48.

[11] T. Ünver, Homogen ve homogen olmayan Cauchy-Riemann denklemleri için parametreye bağllRobin sınır değer problemi, Kırıkkale Uni. J. Adv. Sci., 1 (2012), 58-63.

[12] İ. Gençtürk, The Dirichlet-Neumann boundary value problem for the inhomogeneous Bitsadze equation in a ring domain, Thai J. Math., (in press).

[13] İ. Gençtürk, K. Koca, Neumann boundary value problem for Bitsadze equation in a ring domain, J. Anal., 28 (2020), 799-815.

[14] T. Vaitekhovich, Boundary value problems for complex partial differential equations in a ring domain, Ph.D. Thesis, FU Berlin, 2008.

[15] H. Begehr, Boundary value problems in complex analysis I, Bol. Asoc. Mat. Venezolana, 12(1) (2005), 65-85.

[16] H. Begehr, Boundary value problems in complex analysis II, Bol. Asoc. Mat. Venezolana, 12(2) (2005), $217-250$.

[17] H. Begehr, Complex Analytic Methods for Partial Differential Equations. An Introductory Text, World Scientific, Singapore, 1994. 